\title{
ON PERIODIC MOTIONS OF A TWO DIMENSIONAL TODA TYPE CHAIN*
}

\author{
Gianni MANCINI ${ }^{1}$ AND P.N. SRIKANTH ${ }^{2}$
}

\begin{abstract}
In this paper we consider a chain of strings with fixed end points coupled with nearest neighbour interaction potential of exponential type, i.e.

$$
\left\{\begin{array}{l}
\varphi_{t t}^{i}-\varphi_{x x}^{i}=\exp \left(\varphi^{i+1}-\varphi^{i}\right)-\exp \left(\varphi^{i}-\varphi i-1\right) \quad 0<x<\pi, \quad t \in \mathbb{R}, \mathrm{i} \in \mathbb{Z} \quad \text { (TC) } \\
\varphi^{i}(0, t)=\varphi^{i}(\pi, t)=0 \quad \forall t, i .
\end{array}\right.
$$

We consider the case of "closed chains" i.e. $\varphi^{i+N}=\varphi^{i} \forall i \in \mathbb{Z}$ and some $N \in \mathbb{N}$ and look for solutions which are peirodic in time. The existence of periodic solutions for the dual problem is proved in Orlicz space setting.
\end{abstract}

Mathematics Subject Classification. 58E30, 37J45.

Received July 22, 2003.

\section{InTRODUCTION}

In this paper we consider a chain of strings with fixed end points coupled with nearest neighbor interaction potential of exponential type, i.e.

$$
\left\{\begin{array}{l}
\varphi_{t t}^{i}-\varphi_{x x}^{i}=\exp \left(\varphi^{i+1}-\varphi^{i}\right)-\exp \left(\varphi^{i}-\varphi^{i-1}\right) \quad 0<x<\pi, \quad t \in \mathbb{R}, i \in \mathbb{Z} \\
\varphi^{i}(0, t)=\varphi^{i}(\pi, t)=0 \quad \forall t, i
\end{array}\right.
$$

We consider the case of "closed chains" i.e. $\varphi^{i+N}=\varphi^{i} \forall i \in \mathbb{Z}$ and some $N \in \mathbb{N}$ and we look for solutions which are periodic in time. If $N=2$, it reduces to a sine-Gordon equation, which is known to be completely integrable (see [10]) and to possess periodic solutions (see [4]). Henceforth, we will be interested to the case $N \geq 3$.

The study is motivated by the work [10]. However, it was Toda who initiated studies with exponential interaction. More precisely, Toda considered one-dimensional lattices of $N$ particles with exponential interaction. If $V_{i}$ denotes the potential of interaction between the $i$ th and $(i+1)$ th particle, then the equation for the $i$ th particle reads

$$
\ddot{\varphi}^{i}=V_{i-1}^{\prime}\left(\varphi^{i-1}-\varphi^{i}\right)-V_{i}^{\prime}\left(\varphi^{i}-\varphi^{i+1}\right) .
$$

Keywords and phrases. Periodic, Toda type chain.

* The author's research is supported by MURST under the national project Variational methods and nonlinear differential equations.

1 Dipartimento di Mathematica, Universita di Roma Tre, Via S. Leonardo Murialdo 1, Roma, Italy;

e-mail: mancini@matrm3.mat.uniroma3.it

2 TIFR Centre, IISc Campus, PB 1234, Bangalore - 560 012, India; e-mail: srikanth@math.tifrbng.res.in 
In case $V_{i}(t)=V(t)=\frac{a}{b} \mathrm{e}^{b t}+a t \forall i=1, \ldots, N$ and $a b>0$ one has the classical Toda lattice, which was shown to be completely integrable, with explicit periodic and soliton solutions [14]. Furthermore, it is known by methods of Kolmogorov-Arnold-Moser (KAM) theory [2,9] that periodic and quasi-periodic motions of (T) persist under small perturbations. It is in such a context that periodic motions of lattices has assumed its significance. In [13] Toda type lattices under large perturbations have been studied and existence of periodic solutions has been established. See [5] and [6] for a more complete study of one dimensional lattices.

The problem under consideration in this paper can be thought of as a 2-dimensional generalization of the Toda chain. As far as we know the only work dealing with this kind of problem is [10] where integrability is shown using inverse-problem method. However there is no information about the nature of solutions. Our effort at this stage is to explore if global methods of nonlinear analysis can be employed to study the higher dimensional lattices of Toda type. We plan to takeup the study of more general potentials of interaction in future work.

Returning to (TC), note that it possesses (linear) normal modes $\varphi^{i}=\varphi, \varphi_{t t}-\varphi_{x x}=0$, and infact the motion is determined up to normal modes, i.e. if $\Phi=\left(\varphi^{1}, \ldots \varphi^{N}\right)$ solves (TC), then it does $\Phi+\Gamma$, where $\Gamma=\left(\gamma^{1}, \ldots \gamma^{N}\right)$ with $\gamma^{i}=\gamma(i=1, \ldots, N)$ and $\gamma_{t t}-\gamma_{x x}=0$ (notice that $\sum_{i=1}^{N} \varphi_{t t}^{i}-\varphi_{x x}^{i}=0$ ). To get rid of normal modes, we will ask $\Phi$ to satisfy the normalization condition $\sum_{i=1}^{N} \varphi^{i} \equiv 0$ and prove the following theorem.

Theorem 1.1. (TC) has infinitely many (normalized) periodic solutions.

\section{A dual Problem in Orlicz SPACES}

Problem (TC) can be written in vector form

$$
\square \Phi+\nabla f(\Phi)=0
$$

where $\Phi=\left(\varphi^{1}, \ldots, \varphi^{N}\right), \square \Phi=\left(\phi_{t t}^{i}-\varphi_{x x}^{i}\right)_{i=1, \ldots N}$ and

$$
\begin{gathered}
f(\eta)=\sum_{i=1}^{N}\left[\exp \left(\eta^{i}-\eta^{i-1}\right)-1\right] \quad \eta \in \mathbb{R}^{N}, \eta^{0}=\eta^{N} \\
\nabla f(\eta)=\left(\exp \left(\eta^{1}-\eta^{N}\right)-\exp \left(\eta^{2}-\eta^{1}\right), \ldots, \exp \left(\eta^{N}-\eta^{N-1}\right)-\exp \left(\eta^{1}-\eta^{N}\right)\right) .
\end{gathered}
$$

Clearly, $\nabla f(t \mathbb{1})=0 \forall t$, where $\mathbb{1}=(1, \ldots, 1)$ and $\nabla f(\eta) \in \mathbb{1}^{\perp}=\left\{\xi \in \mathbb{R}^{N}: \sum_{i=1}^{N} \xi^{i}=0\right\}$.

Basic qualitative properties of the interaction potential $f$, proven in the appendix, are as follows. Let $c_{N}:=\frac{2}{\sqrt{N}(N-1)} ;$ then

$$
\begin{aligned}
& \forall \alpha>0, \quad\langle\nabla f(\eta), \eta\rangle \geq \alpha f(\eta) \quad \forall \eta \in \mathbb{1}^{\perp} \text { with }|\eta| \geq \frac{\alpha+\sqrt{N \alpha}}{c_{N}} \quad \text { (arabic@section.arabic@equation) } \\
& \langle\nabla f(\eta), \eta\rangle \geq c_{N}|\eta|\left[\mathrm{e}^{c_{N}|\eta|}-1\right] \quad \forall \eta \in \mathbb{1}^{\perp} \quad \text { (arabic@section.arabic@equation) } \\
& \left\langle D^{2} f(\eta) \xi, \xi\right\rangle \geq c_{N}^{2} \mathrm{e}^{-\sqrt{2}|\eta|}|\xi|^{2} \quad \forall \eta \in \mathbb{R}^{N}, \quad \forall \xi \in \mathbb{1}^{\perp} . \quad \text { (arabic@section.arabic@equation) }
\end{aligned}
$$

Furthermore, if $A(s)=\mathrm{e}^{s}-s-1, s \geq 0$, then

$$
A\left(c_{N}|\eta|\right) \leq f(\eta) \leq N A(\sqrt{2}|\eta|) \quad \forall \eta \in \mathbb{1}^{\perp} . \quad \text { (arabic@section.arabic@equation) }
$$

The function $A$ so defined is an Orlicz function (or $N$-function; see appendix for basic facts on Orlicz functions and Orlicz spaces used throughout the paper). We denote by $L_{A}=L_{A}\left(\Omega_{n}\right)$ the Orlicz space associated to $A$, 
consisting of measurable functions

$$
\Phi: \Omega_{n} \rightarrow \mathbb{R}^{N} \quad \text { such that } \quad \int_{\Omega_{n}} A(\lambda|\Phi|)<\infty \quad \text { for some } \lambda=\lambda(\Phi)>0
$$

where $\Omega_{n}=[0, \pi] \times\left[0, \frac{2 \pi}{n}\right]$ ( $n$ to be chosen, later, suitably large), i.e. $L_{A}=\left\langle K_{A}\right\rangle$ where $K_{A}=\left\{\Phi: \int_{\Omega_{n}} A(|\Phi|)<\right.$ $\infty\} \cdot L_{A}$, endowed with the Orlicz-Luxemburg norm

$$
\|\Phi\|_{A}=\inf \left\{k>0: \int_{\Omega_{n}} A\left(\frac{|\Phi|}{k}\right) \leq 1\right\}
$$

is a Banach space. Thanks to (2.4), one could seek solutions of $(T C)$ by looking for critical points of

$$
\Phi \rightarrow \int_{\Omega_{n}} f(\Phi)-\frac{1}{2} \int_{\Omega_{n}}\left|\Phi_{t}\right|^{2}-\left|\Phi_{x}\right|^{2}
$$

in some Orlicz-Sobolev space associated to $A$ (in the spirit of the celebrated paper [12]). Instead, because of the strong indefiniteness of the quadratic part, we prefer, following [4], to work with a dual functional, via a Legendre transformation. To this end, we first derive some properties of the dual potential $f^{*}$ :

$$
f^{*}(\xi):=\sup _{\eta \in \mathbb{1}^{\perp}}[\langle\xi, \eta\rangle-f(\eta)] \quad \forall \xi \in \mathbb{1}^{\perp} .
$$

Since $\left.f\right|_{\mathbb{1}^{\perp}}$ has superlinear growth and is strictly convex by (2.1)-(2.4), $f^{*}$ turns out to be a $C^{\infty}$-strictly convex function on $\mathbb{1}^{\perp}$. Also, $\nabla f(\eta)=\xi \Leftrightarrow \eta=\nabla f^{*}(\xi)$ and $f^{*}(\xi)=\langle\xi, \eta\rangle-f(\eta)$ for $\xi=\nabla f(\eta)$ and we have

$$
\text { (Young's inequality) } \quad\langle\xi, \eta\rangle \leq f^{*}(\xi)+f(\eta) \quad \forall \xi, \eta \in \mathbb{1}^{\perp} .
$$

We denote by $A^{*}$ the $N$-function complementary to $A$, i.e.

$$
A^{*}(s)=\sup _{t \geq 0}(s t-A(t)), \quad s \geq 0 .
$$

Easily, $A^{*}(s)=(1+s) \log (1+s)-s$, and, by (2.4) and direct computation, we have

Lemma 1. $N A^{*}\left(\frac{|\xi|}{N \sqrt{2}}\right) \leq f^{*}(\xi) \leq A^{*}\left(\frac{|\xi|}{c_{N}}\right) \quad \forall \xi \in \mathbb{1}^{\perp}$.

A crucial feature of $A^{*}$ is that it satisfies a (global) $\left(\Delta_{2}\right)$ property:

$$
\left(\Delta_{2}\right) \quad \forall r>0 \quad \exists k(r)>0: \quad A^{*}(r t) \leq k(r) A^{*}(t) \quad \forall t \geq 0
$$

with $k(r)=\max \left\{r, r^{2}\right\}$. From $\left(\Delta_{2}\right)$ and Lemma 1 it follows

$$
\frac{1}{2 N} A^{*}(|\xi|) \leq f^{*}(\xi) \leq \frac{1}{c_{N}^{2}} A^{*}(|\xi|) \quad \forall \xi \in \mathbb{1}^{\perp} . \quad \text { (arabic@section.arabic@equation) }
$$

Hence, the natural space to work in is the Orlicz space $L_{A^{*}}$. Note that, thanks to $\left(\Delta_{2}\right), L_{A^{*}}=K_{A^{*}}$. In particular, $L_{A^{*}}$ is a separable Banach space (see Prop. A1). Also, from Hölder's inequality

$$
\int\langle\Phi, \Psi\rangle \leq N\|\Phi\|_{A}\|\Psi\|_{A^{*}} \quad \forall \Phi \in L_{A}, \Psi \in L_{A^{*}},
$$

it follows that every $\Phi \in L_{A}$ defines an element of $L_{A^{*}}^{\prime}$ and infact, since $A^{*}$ satisfies $\left(\Delta_{2}\right), L_{A^{*}}^{\prime}$ turns out to be isomorphic and homeomorphic to $L_{A}$. 
We now proceed to establish some properties of the functional $F(\Psi):=\int_{\Omega_{n}} f^{*}(\Psi)$, and its "gradient operator" $\Psi \rightarrow \nabla f^{*}(\Psi)$, on $\mathrm{E}_{A^{*}}:=\left\{\Psi \in L_{A^{*}}: \sum_{i=1}^{N} \psi^{j} \equiv 0\right\}$.

\section{Lemma 2.}

(i) $\left\|\nabla f^{*}(\Psi)\right\|_{A} \leq \frac{1}{c_{N}}\left(1+\frac{\|\Psi\|_{A^{*}}^{2}}{c_{N}^{2}}\right) \quad \forall \Psi \in E_{A^{*}}$.

(ii) $\left\|\Psi_{j}-\Psi\right\|_{A^{*}} \rightarrow 0 \Rightarrow \int_{\Omega_{n}}\left\langle\nabla f^{*}\left(\Psi_{j}\right), \hbar\right\rangle \rightarrow \int_{\Omega_{n}}\left\langle\nabla f^{*}(\Psi), \hbar\right\rangle \quad \forall \hbar \in L_{A^{*}}$.

(iii) $F(\Psi)=\int_{\Omega_{n}} f^{*}(\Psi)$ is a locally Lipschitz functional on $E_{A^{*}}$.

(iv) $\Psi \rightarrow \nabla f^{*}(\Psi)$ is the Gateaux derivative of F. i.e.

$$
\frac{\mathrm{d}}{\mathrm{d} t}\left[\int_{\Omega_{n}} f^{*}(\Psi+t \hbar)\right]_{\mid t=0}=\int_{\Omega_{n}}\left\langle\nabla f^{*}(\Psi), \hbar\right\rangle \quad \forall \Psi, \hbar \in E_{A^{*}} .
$$

Proof. (i) Let $\Phi=\nabla f^{*}(\Psi)$, and hence $\Psi=\nabla f(\Phi)$. From (2.2) we see that

$$
\frac{1}{c_{N}}|\Psi| \geq \mathrm{e}^{c_{N}|\Phi|}-1
$$

and hence, by monotonicity,

$$
A^{*}\left(\frac{|\Psi|}{c_{N}}\right) \geq A^{*}\left(\mathrm{e}^{c_{N}|\Phi|}-1\right)=c_{N}|\Phi| \mathrm{e}^{c_{N}|\Phi|}-\mathrm{e}^{c_{N}|\Phi|}+1 \geq A\left(c_{N}|\Phi|\right) .
$$

Since $\int_{\Omega_{n}} A^{*}(|\Psi|) \leq \sup _{t>0} \frac{A^{*}\left(\|\Psi\|_{A *} t\right)}{A^{*}(t)} \leq \max \left\{\|\Psi\|_{A^{*}},\|\Psi\|_{A *}^{2}\right\} \quad \forall \Psi \in L_{A^{*}}$, (see Prop. A2 (ii) and $\left(\Delta_{2}\right)$ for $A^{*}$ ), then

$$
\int_{\Omega_{n}} A\left(c_{N}\left|\nabla f^{*}(\Psi)\right|\right) \leq 1+\frac{\|\Psi\|_{A^{*}}^{2}}{c_{N}^{2}} \quad \forall \Psi \in \mathrm{E}_{A^{*}} .
$$

Finally, using Proposition A2 (i), we obtain

$$
\left\|c_{N} \nabla f^{*}(\Psi)\right\|_{A} \leq 1+\frac{\|\Psi\|_{A^{*}}^{2}}{c_{N}^{2}} \quad \forall \Psi \in \mathrm{E}_{A^{*}}
$$

(ii) From $\left\|\Psi_{j}-\Psi\right\|_{A^{*}} \rightarrow 0$, we have $\int A^{*}\left(\left|\Psi_{j}-\Psi\right|\right) \rightarrow 0$ (see Prop. A2 (i)) and hence $\Psi_{j} \rightarrow \Psi$ in measure. In addition, we know by (i) that $\left\|\nabla f^{*}\left(\Psi_{j}\right)\right\|_{A}$ is bounded and hence (ii) follows by Proposition A3.

(iii) Let $\Psi_{1}, \Psi_{2} \in B_{r}(0)$. Then

$$
\begin{aligned}
\left|F\left(\Psi_{1}\right)-F\left(\Psi_{2}\right)\right| & =\left|\int_{\Omega_{n}} \int_{0}^{1}\left\langle\nabla f^{*}\left(t \Psi_{1}+(1-t) \Psi_{2}\right), \Psi_{1}-\Psi_{2}\right\rangle\right| \\
& \leq N\left\|\Psi_{1}-\Psi_{2}\right\|_{A^{*}} \sup _{\Psi \in B_{r}(0)}\left\|\nabla f^{*}(\Psi)\right\|_{A}
\end{aligned}
$$

with $\sup _{\Psi \in B_{r}(0)}\left\|\nabla f^{*}(\Psi)\right\|_{A}<\infty$ by (i).

(iv) Follows from $\int_{\Omega_{n}} \frac{f^{*}(\bar{\Psi}+t \hbar)-f^{*}(\bar{\Psi})}{t}=\int_{0}^{1}\left(\int_{\Omega_{n}}\left\langle\nabla f^{*}(\bar{\Psi}+\tau t \hbar), \hbar\right\rangle\right) \mathrm{d} \tau$ and (see (ii))

$$
\left.\int_{\Omega_{n}}\left\langle\nabla f^{*}(\bar{\Psi})+\tau t \hbar\right), \hbar\right\rangle \rightarrow \int_{\Omega_{n}}\left\langle\nabla f^{*}(\bar{\Psi}), \hbar\right\rangle \quad \forall \tau \in[0,1],
$$

because, by (i),

$$
\left|\int_{\Omega_{n}}\left\langle\nabla f^{*}(\bar{\Psi}+\tau t \hbar), \hbar\right\rangle\right| \leq\|\hbar\| \sup _{\|\bar{\Psi}-\Psi\|_{A^{*}} \leq\|\hbar\|_{A^{*}}}\left\|\nabla f^{*}(\Psi)\right\| \leq c .
$$


Let us now recall basic properties of the operator $\square=\frac{\partial^{2}}{\partial t^{2}}-\frac{\partial^{2}}{\partial x^{2}}$ acting on summable functions which are $2 \pi$ periodic and satisfy Dirichlet boundary conditions. It is known (see $[4,8]$ ) that $\square \gamma=0$ (in the weak sense), if and only if

$$
\gamma(x, t)=p(x+t)-p(t-x)
$$

for some $p \in L_{l o c}^{1}(\mathbb{R}), 2 \pi$-periodic and such that $\int_{0}^{2 \pi} p=0$. Also, for a given summable $\psi$, we have that $\int_{[0, \pi] \times[0,2 \pi]} \psi \gamma=0 \quad \forall \gamma \in L^{\infty} \cap \operatorname{Ker} \square$ if and only if

$$
\int_{0}^{\pi}[\psi(x, t-x)-\psi(x, t+x)] \mathrm{d} x=0 \quad \text { for a.e. } t \quad \text { (arabic@section.arabic@equation) }
$$

and, for such a $\psi$, there is a unique $2 \pi$ periodic continuous function $\varphi=L \psi$ satisfying the boundary condition $\varphi(0, t)=\varphi(\pi, t)=0 \forall t$, such that

$$
\square \varphi=\psi \quad \text { and } \quad \int_{[0, \pi] \times[0,2 \pi]} \psi \gamma=0 \quad \forall \gamma \in \operatorname{Ker} \square \cap L^{\infty} .
$$

Actually, $L \psi$ is explicitely known (see [8]):

$$
L \psi(x, t)=\tilde{\varphi}+p(t+x)-p(t-x)
$$

where

and

$$
\tilde{\varphi}(x, t)=-\frac{1}{2} \int_{x}^{\pi} \mathrm{d} \xi \int_{t+x-\xi}^{t-x+\xi} \psi(\xi, \tau) \mathrm{d} \tau+\frac{\pi-x}{2 \pi} \int_{0}^{\pi} \mathrm{d} \xi \int_{t-\xi}^{t+\xi} \psi(\xi, \tau) \mathrm{d} \tau
$$

It can be easily checked that

$$
p(y)=\frac{1}{2 \pi} \int_{0}^{\pi}[\tilde{\varphi}(s, y-s)-\tilde{\varphi}(s, y+s)] \mathrm{d} s .
$$

$$
\begin{array}{cll}
\exists C>0: & \|L \psi\|_{L^{\infty}} \leq C\|\psi\|_{L^{1}}, \quad\|L \psi\|_{C^{0,1}} \leq C\|\psi\|_{L^{\infty}} & \text { (arabic@section.arabic@equation) } \\
\psi \in C^{k, 1} \Rightarrow L \psi \in C^{k+1,1} . & \text { (arabic@section.arabic@equation) }
\end{array}
$$

Let us now write $\quad \mathbb{K}:=(\operatorname{Ker} \square)^{N}, \quad L \Psi=\left(L \psi^{1}, \ldots, L \psi^{N}\right)$, and set

$$
E=\left\{\Psi \in L_{A^{*}}: \quad \sum_{j=1}^{N} \psi^{j} \equiv 0, \quad \int_{\Omega_{n}}\langle\Psi, \Gamma\rangle=0 \quad \forall \Gamma \in \mathbb{K} \cap L_{A}\right\} .
$$

Note that $E$ is a closed subspace of $\mathrm{E}_{A^{*}}$ and is a separable Banach space.

Lemma 3. $L$ induces a compact linear operator from $E$ into $L_{A}$.

Proof. Since $L_{A^{*}} \subset L^{1}, L \Psi$ is infact in $L^{\infty}$. Let $\Psi_{k}$ be a bounded sequence in $E$. Since $\sup _{k}\left\|\Psi_{k}\right\|_{A^{*}}<\infty$ implies $\sup _{k}\left\|\Psi_{k}\right\|_{L^{1}}<\infty, L \Psi_{k}$ is bounded in $L^{\infty}$. We want to prove $L \Psi_{k}$ are equicontinuous and Ascoli-Arzela will imply compactness. Using the notations introduced above,

$$
L \Psi_{k}=\tilde{\Phi}_{k}+P_{k}(t+x)-P_{k}(t-x), \quad P=\left(p^{i}\right)
$$

it is clearly enough to prove that $\tilde{\Phi}_{k}$ are equicontinuous. We have (dropping superscripts)

$$
\left|\tilde{\varphi}_{k}(x, t)-\tilde{\varphi}_{k}\left(x^{\prime}, t^{\prime}\right)\right| \leq \frac{1}{2}\left|\int_{x}^{\pi} \mathrm{d} \xi \int_{t+x-\xi}^{t-x+\xi} \psi_{k}(\xi, \tau) \mathrm{d} \tau-\int_{x^{\prime}}^{\pi} \mathrm{d} \xi \int_{t^{\prime}+x^{\prime}-\xi}^{t^{\prime}-x^{\prime}+\xi} \psi_{k}(\xi, \tau) \mathrm{d} \tau\right|+\frac{c_{k}}{2 \pi}\left|x^{\prime}-x\right|
$$


where $c_{k}:=\int_{0}^{\pi} \mathrm{d} \xi \int_{t-\xi}^{t+\xi} \psi_{k}(\xi, \tau) \mathrm{d} \tau$ are constants bounded by $\sup _{k}\left\|\psi_{k}\right\|_{L^{1}}$ (recall (2.6)). Hence it is enough to estimate

$$
\Delta=\left|\int_{T_{x, t}} \psi_{k}(\xi, \tau) \mathrm{d} \xi \mathrm{d} \tau-\int_{T_{x^{\prime}, t^{\prime}}} \psi_{k}(\xi, \tau) \mathrm{d} \xi \mathrm{d} \tau\right| \leq \int_{\left(T_{x, t} \backslash T_{x^{\prime}, t^{\prime}}\right) \cup\left(T_{x^{\prime} t^{\prime}} \backslash T_{x, t}\right)}\left|\psi_{k}\right|
$$

where

$$
T_{x, t}=\{(\xi, \tau) ; x \leq \xi \leq \pi, \quad t+x-\xi \leq \tau \leq t-x+\xi\} \subset[0, \pi] \times \mathbb{R}
$$

and $\psi_{k}$ are extended as periodic functions on $[0, \pi] \times \mathbb{R}$. Clearly the measure of the symmetric difference is small if $(x, t)$ and $\left(x^{\prime}, t^{\prime}\right)$ are close. On the other hand by Vallée-Poussin theorem [7] (p. 103)

$$
\sup _{k} \int_{\Omega_{n}} A^{*}\left(\left|\psi_{k}\right|\right)<+\infty \Rightarrow \sup _{k} \int_{\Sigma}\left|\psi_{k}\right| \leq \varepsilon \quad \text { if }|\Sigma| \leq \delta_{\varepsilon}
$$

Since $\int A^{*}\left(\left|\Psi_{k}\right|\right) \leq 1+\left\|\Psi_{k}\right\|_{A^{*}}^{2}$ (see Prop. A2 (ii) and $\left(\Delta_{2}\right)$ for $A^{*}$ ) and $\sup _{k}\left\|\Psi_{k}\right\|_{A^{*}}<+\infty$, we have

$$
\left|\tilde{\varphi}_{k}(x, t)-\tilde{\varphi}_{k}\left(x^{\prime}, t^{\prime}\right)\right| \leq \varepsilon \quad \text { if } \quad\left|x-x^{\prime}\right|+\left|t-t^{\prime}\right| \leq \delta_{\varepsilon}
$$

uniformly with respect to $k$. Hence the result.

We now state the dual variational principle.

Proposition 4. Let $\Psi$ be a critical point of the (Gateaux differentiable) functional

$$
S(\Psi)=\int_{\Omega_{n}} f^{*}(\Psi)+\frac{1}{2} \int_{\Omega_{n}}\langle\Psi, L \Psi\rangle, \quad \Psi \in E . \quad \text { (arabic@section.arabic@)equation) }
$$

Then $\Phi=\nabla f^{*}(\Psi)$ is a (weak) $\frac{2 \pi}{n}$ periodic solution of $(T C)$.

Proof. Since $\mathbb{K}$ is closed in $L^{1}, \mathbb{K} \cap L_{A}$ is closed in $L_{A}$, as well as $L_{A} \cap \mathbb{K}^{\perp}:=\left\{\Phi \in L_{A}: \int_{\Omega_{n}}\langle\Phi, \Gamma\rangle=0 \quad \forall \Gamma \in\right.$ $\left.\mathbb{I} \cap L_{A^{*}}\right\}$. Thus we can write

$$
\nabla f^{*}(\Psi)+L \Psi=W+\Gamma, \quad W \in \mathbb{K}^{\perp} \cap L_{A}, \quad \Gamma \in \mathbb{K} \cap L_{A}
$$

Notice that $\sum_{j} w^{j}+\gamma^{j} \equiv 0$ and hence we can assume $\sum_{j} w^{j} \equiv 0$. Since, by assumption,

$$
\int_{\Omega_{n}}\left\langle\nabla f^{*}(\Psi), \hbar\right\rangle+\int_{\Omega_{n}} \hbar L \Psi=0 \quad \forall \hbar \in E
$$

we see that $W=0$. Hence $\nabla f^{*}(\Psi)+L \Psi=\Gamma$. Let $\Phi=\nabla f^{*}(\Psi)=\Gamma-L \Psi$. We have

$$
-\square \Phi=\Psi=\nabla f(\Phi)
$$

i.e. $\Phi$ is a $\frac{2 \pi}{n}$ periodic solution of (TC) in the weak sense.

We end this section stating some growth properties of $\nabla f^{*}$ which will be crucial to get critical points of $S$. For a given $\theta<1$, let $m_{\theta}=\sqrt{2}(\alpha+\sqrt{N \alpha})$ where $\alpha=(1-\theta)^{-1}$. Then

\section{Lemma 5.}

(i) $\frac{1}{c_{N}} \log \left(1+\frac{|\xi|}{c_{N}}\right) \geq\left|\nabla f^{*}(\xi)\right| \geq \frac{N}{|\xi|} A^{*}\left(\frac{|\xi|}{N \sqrt{2}}\right) \quad \forall \xi \in \mathbb{1}^{\perp}, \quad \xi \neq 0$.

(ii) $\forall \theta<1, f^{*}(\xi) \geq \theta\left\langle\nabla f^{*}(\xi), \xi\right\rangle$ if $|\xi| \geq c(N, \theta):=4 N \mathrm{e}^{\frac{m_{\theta}}{c_{N}}}$. 
Proof.

(i) From Lemma 1 and $\left\langle\nabla f^{*}(\xi), \xi\right\rangle \geq f^{*}(\xi)$, we obtain the RHS inequality. Next, if $\eta=\nabla f^{*}(\xi)$, from (2.2) we derive $|\xi|=|\nabla f(\eta)| \geq c_{N}\left[\mathrm{e}^{c_{N}|\eta|}-1\right]=c_{N}\left[\mathrm{e}^{c_{N}\left|\nabla f^{*}(\xi)\right|}-1\right]$. This gives the LHS inequality.

(ii) From the RHS inequality in (i), one easily obtains

$$
|\xi| \geq 4 N \mathrm{e}^{\frac{m_{\theta}}{c_{N}}} \Rightarrow\left|\nabla f^{*}(\xi)\right| \geq \frac{\alpha+\sqrt{N \alpha}}{c_{N}} .
$$

Hence (ii) follows from $(2.1)$ and $f^{*}(\xi)=\left\langle\nabla f^{*}(\xi), \xi\right\rangle-f(\eta), \eta=\nabla f^{*}(\xi)$.

\section{EXISTENCE OF WEAK PERIODIC SOLUTIONS}

In this section we will prove our theorem, by means of a suitable version of the Mountain Pass Lemma.

Lemma ([4]). Let $E$ be a separable Banach space and let $S: E \rightarrow \mathbb{R}$ be a locally Lipschitz Gateaux differentiable functional. Suppose further

(i) $\Psi_{j} \rightarrow \Psi \Rightarrow S^{\prime}\left(\Psi_{j}\right) \hbar \rightarrow S^{\prime}(\Psi) \hbar \quad \forall \hbar \in E$;

(ii) $\exists U$ open, $0 \in U$ such that $\inf _{\partial U} S>S(0)$;

(iii) $\exists \Psi_{0} \notin U$ such that $S\left(\Psi_{0}\right) \leq S(0)$;

(iv) $S\left(\Psi_{j}\right) \rightarrow c, S^{\prime}\left(\Psi_{j}\right) \rightarrow 0 \Rightarrow \exists \Psi \in S^{-1}(c)$ such that $S^{\prime}(\Psi)=0$.

Then

$$
c:=\inf _{p \in \mathbb{P}} \max _{t \in[0,1]} S(p(t))
$$

is a critical value, where $\mathbb{P}=\left\{p \in C([0,1], E): p(0)=0, p(1)=\Psi_{0}\right\}$.

We have already seen that $S$ defined by $(2.9)$ is locally Lipschitz and Gateaux differentiable, with derivative given by $S^{\prime}(\Psi) \hbar=\int\left\langle\nabla f^{*}(\Psi), \hbar\right\rangle+\langle L \Psi, \hbar\rangle, \forall \hbar \in E$. We now prove $S$ satisfies (i)-(iv).

Proof of (i). It follows by Lemma 2 (iii) and continuity properties of $L$.

Proof of (ii). It is exactly here where we need to take $n$ suitably large. Let $U=\left\{\Psi \in E: \int_{\Omega_{n}} f^{*}(\Psi)<2 N\left|\Omega_{n}\right|\right\}$. From Young's inequality and $\max _{|\xi|=1} f<2 N$, we get

$$
|\xi| \leq f^{*}(\xi)+f\left(\frac{\xi}{|\xi|}\right) \leq 2 N+f^{*}(\xi) \quad \forall \xi \in \mathbb{1}^{\perp}
$$

and hence $\int_{\Omega_{n}}\left|\psi^{j}\right| \leq 2 N\left|\Omega_{n}\right|+\int_{\Omega_{n}} f^{*}(\Psi)=4 N\left|\Omega_{n}\right| \quad \forall j$ and $\Psi \in \partial U$. Since $\int_{\Omega_{n}}\langle\Psi, L \Psi\rangle \geq-2 \sum_{j=1}^{N}\left(\int_{\Omega_{n}}\left|\psi^{j}\right|\right)^{2}$ (see [4]) we have, for $\Psi \in \partial U$,

$$
S(\Psi) \geq 2 N\left|\Omega_{n}\right|-\sum\left(\int_{\Omega_{n}}\left|\psi^{j}\right|\right)^{2} \geq 2 N\left|\Omega_{n}\right|-16 N^{3}\left|\Omega_{n}\right|^{2}>0=S(0) \text { if } n>16 \pi^{2} N^{2} .
$$

Proof of (iii). It follows from the subquadratic behaviour of $f^{*}$ at infinity.

Proof of (iv). Let $\Psi_{j}$ be such that

$$
\int_{\Omega_{n}} f^{*}\left(\Psi_{j}\right)+\frac{1}{2} \int_{\Omega_{n}}\left\langle\Psi_{j}, L \Psi_{j}\right\rangle=c+\circ(1) \text { and } \sup _{\substack{\|\hbar\|_{A^{*} \leq 1} \\ \hbar \in E}}\left|\int_{\Omega_{n}}\left\langle\nabla f^{*}\left(\Psi_{j}\right)+L \Psi_{j}, \hbar\right\rangle\right|=\circ(1) \text { as } j \rightarrow \infty
$$

and hence

$$
2 \int_{\Omega_{n}} f^{*}\left(\Psi_{j}\right)-\int_{\Omega_{n}}\left\langle\nabla f^{*}\left(\Psi_{j}\right), \Psi_{j}\right\rangle=2 c+\circ(1)+\circ(1)\left\|\Psi_{j}\right\|_{A^{*}}
$$


By Lemma 5 (ii) and $f^{*} \geq 0$, we have

$$
\int_{\Omega_{n}} f^{*}\left(\Psi_{j}\right) \geq \theta \int_{\Omega_{n}}\left\langle\nabla f^{*}\left(\Psi_{j}\right), \Psi_{j}\right\rangle-\theta \tilde{c}(N, \theta)
$$

where $\tilde{c}(N, \theta)=\left[\frac{1}{c_{N}} \log \left(1+\frac{c(\theta, N)}{c_{N}}\right)\right]\left|\Omega_{n}\right|$. Hence

$$
\circ(1)\left\|\Psi_{j}\right\|_{A^{*}} \geq\left(2-\frac{1}{\theta}\right) \int_{\Omega_{n}} f^{*}\left(\Psi_{j}\right)-[\tilde{c}(N, \theta)+2 c+\circ(1)] . \quad \text { (arabic@section.arabic@equation) }
$$

From (2.5) and Proposition A2 (i), we have

$$
\int_{\Omega_{n}} f^{*}\left(\Psi_{j}\right) \geq \frac{1}{2 N}\left[\left\|\Psi_{j}\right\|_{A^{*}}-1\right] . \quad \text { (arabic@section.arabic@equation) }
$$

Taking $\theta>\frac{3}{4}$, we obtain from (3.1) and (3.2)

$$
\frac{1}{3 N}\left\|\Psi_{j}\right\|_{A^{*}} \leq \frac{1}{2 N}+\tilde{c}(N, \theta)+2 c . \quad \text { (arabic@section.arabic@equation) }
$$

Hence $\sup _{j}\left\|\Psi_{j}\right\|_{A^{*}}<\infty$. Then (see [7] 14.4) $\exists \Psi \in E$ such that, for a subsequence,

$$
\int_{\Omega_{n}}\langle\Psi, \Phi\rangle \rightarrow \int_{\Omega_{n}}\langle\Psi, \Phi\rangle \quad \forall \Phi \in E_{A}
$$

where $E_{A}$ is the closure of the set of bounded functions in $L_{A}$. We want to show that

$$
\int_{\Omega_{n}}\left\langle\nabla f^{*}(\Psi)+L \Psi, \hbar\right\rangle=0 \quad \forall \hbar \in E
$$

i.e. $\Psi$ is a critical point at level $c$. We will use here, following [4], the so called Minty-trick, exploiting monotonicity of the operator $\Psi \rightarrow \nabla f^{*}(\Psi)$ :

$$
\int\left\langle\nabla f^{*}\left(\Psi_{j}\right)-\nabla f^{*}(\hbar), \quad \Psi_{j}-\hbar\right\rangle \geq 0 \quad \forall \hbar \in L_{A^{*}} . \quad \text { (arabic@section.arabic@equation) }
$$

Writing

$$
\nabla f^{*}\left(\Psi_{j}\right)+L \Psi_{j}=W_{j}+\Gamma_{j}, \quad W_{j} \in L_{A} \cap \mathbb{K}^{\perp}, \Gamma_{j} \in L_{A} \cap \mathbb{K}
$$

with $\sum_{i} w_{j}^{i}=0$ and $\left\|W_{j}\right\|_{A} \rightarrow 0$ (see Prop. 4) and inserting in (3.4), we get

$$
\int\left\langle W_{j}-L \Psi_{j}-\nabla f^{*}(\hbar), \Psi_{j}-\hbar\right\rangle \geq 0 \quad \forall \hbar \in L_{A^{*}} . \quad \text { (arabic@section.arabic@equation) }
$$

If $\hbar \in L^{\infty}$, we can pass to the limit in (3.5), to get

$$
\int\left\langle L \Psi+\nabla f^{*}(\hbar), \Psi-\hbar\right\rangle \leq 0, \quad \forall \hbar \in L^{\infty} . \quad \text { (arabic@section.arabic@sequation) }
$$

Now, choose as test function ${ }_{n} \Psi-t \hbar, \hbar \in L^{\infty}$ where

$$
{ }_{n} \Psi=\left({ }_{n} \psi^{j}\right), \quad{ }_{n} \psi=\left\{\begin{array}{lll}
\psi & \text { if } & |\psi| \leq n \\
0 & \text { if } & |\psi|>n
\end{array}\right.
$$


Then we have

$$
\int\left\langle L \Psi+\nabla f^{*}\left({ }_{n} \Psi-t \hbar\right),\left(\Psi-{ }_{n} \Psi\right)+t \hbar\right\rangle \leq 0
$$

Since $A^{*}$ is $\Delta_{2},\left\|_{n} \Psi-\Psi\right\|_{A^{*}} \rightarrow 0$ (see [7] 10.1) and hence

$$
\int\left\langle L \Psi+\nabla f^{*}(\Psi-t \hbar), t \hbar\right\rangle \leq 0 \quad \forall \hbar \in L^{\infty}, \quad \text { (arabic@section.arabic@equation) }
$$

by Lemma 2 (ii) and because $\nabla f^{*}\left({ }_{n} \Psi-t \hbar\right)$ is bounded in $L_{A}$ by Lemma 2 (i). Now, since $L^{\infty}$ is dense in $L_{A^{*}}$, arguments as above give

$$
\int\left\langle L \Psi+\nabla f^{*}(\Psi-t \hbar), \hbar\right\rangle \leq 0 \quad \forall \hbar \in E \text { and } t>0
$$

Letting $t \rightarrow 0$, we get

$$
\int\left\langle L \Psi+\nabla f^{*}(\Psi), \hbar\right\rangle=0 \quad \forall \hbar
$$

The theorem now follows from Lemma [4]. We remark that the existence of infinitely many solutions follows from the simple device (see [11]) of working in a space of functions whose period is smaller than the minimal period of the solution previously obtained.

\section{Regularity}

Since solutions to (TC) are determined up to normal modes, no regularity result is available for arbitrary weak solutions. Still, following some ideas in [3], and heavily exploiting the periodic structure of the lattice, we will prove that normalized weak solutions are $C^{\infty}$. Actually, we will develope a bootstrap argument for $\Psi=\left(\psi^{j}\right), \Gamma=\left(\gamma^{j}\right)$ satisfying

$$
\begin{array}{r}
\Gamma \in \operatorname{Ker} \square \Psi \in L^{1}, \quad \int \Psi \Pi=0 \forall \Pi \in L^{\infty} \cap \operatorname{Ker} \square \quad \text { (arabic@section.arabic@sequation) } \\
\Psi=\nabla f(\Gamma-L \Psi)
\end{array}
$$

which will lead to $C^{\infty}$ regularity for $\Psi$. Clearly, $\Phi:=\Gamma-L \Psi$ is a weak solution of (TC) and, if $\sum \varphi^{j} \equiv 0$, then $\Phi=\nabla f^{*}(\Psi) \in C^{\infty}$. We start with a simple summability lemma.

Lemma 6. $\nabla f(\Phi) \in L^{1} \Rightarrow \int_{\Omega_{n}} \exp \left(\varphi^{j}-\varphi^{j-1}\right)<\infty \quad \forall j$.

Proof. Arguing by contradiction, we find $j$ such that $\int_{\Omega_{n}} \exp \left[\varphi^{j}-\varphi^{j-1}\right]=+\infty$ and hence $A_{0}:=\left\{\varphi^{j}-\varphi^{j-1} \geq 1\right\}$ has positive measure. Since $\exp \left[\varphi^{j}-\varphi^{j-1}\right]-\exp \left[\varphi^{j+1}-\varphi^{j}\right]=\psi^{j} \in L^{1}$ we have $\int_{A_{0}} \exp \left(\varphi^{j+1}-\varphi^{j}\right)=+\infty$ and hence $A_{1}:=A_{0} \cap\left\{\varphi^{j+1}-\varphi^{j} \geq 1\right\}$ has positive measure. After $N$ iterations, we find $\varphi^{j+i} \geq \varphi^{j+i-1}+1$ for $i=0, \ldots, N-1$ on a set of positive measure $A_{N-1}$. Adding up these inequalities we find a contradiction.

Remark. If $\Phi$ is a normalized weak solution with $\nabla f(\Phi) \in L^{1}$, then, by the previous lemma and Lemma A1, we have $\int \mathrm{e}^{c_{N}|\Phi|}<\infty$. Even this simple summability property fails for arbitrary weak solutions.

To start the bootstrap argument, we first need a couple of lemmata.

Lemma 7. Let $\Psi, \Gamma$ satisfy (4.1). Then $\gamma^{j}-\gamma^{j-1} \in L^{\infty} \forall j$.

Proof. First, $\tilde{\Phi}:=-L \Psi$ belongs to $L^{\infty}$ by $(2.7)$. Let $M:=2\|\tilde{\Phi}\|_{\infty}$. Denoted $\Phi:=\Gamma+\tilde{\Phi}$, it results $\gamma^{j}-\frac{M}{2} \leq$ $\varphi^{j} \leq \gamma^{j}+\frac{M}{2}$. Also, by assumption,

$$
\psi^{j}=\exp \left[\gamma^{j}-\gamma^{j-1}+\tilde{\varphi}^{j}-\tilde{\varphi}^{j-1}\right]-\exp \left[\gamma^{j+1}-\gamma^{j}+\tilde{\varphi}^{j+1}-\tilde{\varphi}^{j}\right] \quad \text { (arabic@section.arabic@equation) }
$$


satisfy (2.6). We have

$$
\begin{aligned}
\exp \left(\gamma^{j}-\gamma^{j-1}-M\right)-\exp \left(\gamma^{j+1}-\gamma^{j}+M\right) & \leq \exp \left(\varphi^{j}-\varphi^{j-1}\right)-\exp \left(\varphi^{j+1}-\varphi^{j}\right) \\
& \leq \exp \left(\gamma^{j}-\gamma^{j-1}+M\right)-\exp \left(\gamma^{j+1}-\gamma^{j}-M\right)
\end{aligned}
$$

(arabic@s section.arabic@̣

Let $\gamma^{j}=p^{j}(t+x)-p^{j}(t-x), \gamma^{j}-\gamma^{j-1}=\tilde{p}^{j}(t+x)-\tilde{p}^{j}(t-x)$, where $\tilde{p}^{j}:=p^{j}-p^{j-1}$, and rewrite (4.3) as

$$
\begin{aligned}
& \exp \left[\tilde{p}^{j}(t+x)-\tilde{p}^{j}(t-x)-M\right]-\exp \left[\tilde{p}^{j+1}(t+x)-\tilde{p}^{j+1}(t-x)+M\right] \leq \psi^{j}(x, t) \\
& \leq \exp \left[\tilde{p}^{j}(t+x)-\tilde{p}^{j}(t-x)+M\right]-\exp \left[\tilde{p}^{j+1}(t+x)-\tilde{p}^{j+1}(t-x)-M\right]
\end{aligned}
$$

Computing at $(x, t-x),(x, t+x)$ respectively and subtracting, we get

$$
\begin{aligned}
& \exp \left[\tilde{p}^{j}(t)-\tilde{p}^{j}(t-2 x)-M\right]-\exp \left[\tilde{p}^{j+1}(t)-\tilde{p}^{j+1}(t-2 x)+M\right] \\
& +\exp \left[\tilde{p}^{j+1}(t+2 x)-\tilde{p}^{j+1}(t)-M\right]-\exp \left[\tilde{p}^{j}(t+2 x)-\tilde{p}^{j}(t)+M\right] \leq \psi^{j}(x, t-x)-\psi^{j}(x, t+x) \\
& \quad \leq \exp \left[\tilde{p}^{j}(t)-\tilde{p}^{j}(t-2 x)+M\right]-\exp \left[\tilde{p}^{j+1}(t)-\tilde{p}^{j+1}(t-2 x)-M\right]
\end{aligned}
$$

$-\exp \left[\tilde{p}^{j}(t+2 x)-\tilde{p}^{j}(t)-M\right]+\exp \left[\tilde{p}^{j+1}(t+2 x)-\tilde{p}^{j+1}(t)+M\right] \cdot \quad$ (arabic@section.arabic@equation)

Notice that, due to Lemma $6, \sinh \tilde{p}^{j} \in L^{1}([0,2 \pi]) \forall j$. In fact, by Fubini and $2 \pi$-periodicity,

$$
\infty>\int_{0}^{2 \pi} \int_{0}^{\pi} \mathrm{e}^{\tilde{p}^{j}(t+x)-\tilde{p}^{j}(t-x)} \mathrm{d} x \mathrm{~d} t=\int_{0}^{2 \pi}\left(\mathrm{e}^{\tilde{p}^{j}(s)} \int_{0}^{\pi} \mathrm{e}^{-\tilde{p}^{j}(s-2 x)} \mathrm{d} x\right) \mathrm{d} s=\frac{1}{2} \int_{0}^{2 \pi} \mathrm{e}^{\tilde{p}^{j}(s)} \mathrm{d} s \int_{0}^{2 \pi} \mathrm{e}^{-\tilde{p}^{j}(\tau)} \mathrm{d} \tau .
$$

Integrating w.r. to $x \in[0, \pi]$ the inequalities (4.4) and changing variables $s=t-2 x$ and $s=t+2 x$, we get, using $2 \pi$-periodicity of $\tilde{p}^{j}$ and property $(2.6)$ of $\psi^{j}$, we obtain

$$
\begin{array}{r}
\int_{0}^{2 \pi}\left[\sinh \left(\tilde{p}^{j}(t)-\tilde{p}^{j}(s)-M\right)+\sinh \left(\tilde{p}^{j+1}(s)-\tilde{p}^{j+1}(t)-M\right)\right] \mathrm{d} s \leq 0 \text { for a.e. } t \\
\quad \text { (arabic@section.arabic@equation) } \\
\int_{0}^{2 \pi}\left[\sinh \left(\tilde{p}^{j}(t)-\tilde{p}^{j}(s)+M\right)+\sinh \left(\tilde{p}^{j+1}(s)-\tilde{p}^{j+1}(t)+M\right)\right] \mathrm{d} s \geq 0, \quad \text { for a.e. } t . \\
\text { (arabic@section.arabic@sequation) }
\end{array}
$$

We now derive from (4.5) that ess $\sup \tilde{p}^{j}<\infty \forall j$. We argue by contradiction. Assume that $A_{k}^{j}=\left\{t: \tilde{p}^{j}(t) \geq k\right\}$ has positive measure for some $j \in\{1, \ldots N\}$ and every $k \in \mathbb{N}$. We claim that this implies $A_{k}^{j+1}=\left\{t \in A_{k}^{j}\right.$ : $\left.\tilde{p}^{j+1}(t) \geq k\right\}$ has positive measure $\forall k$. If not, $\exists \bar{k}$, and $t_{k} \in A_{k}^{j} \subset A_{\bar{k}}^{j} \forall k \geq \bar{k}$ such that $\tilde{p}^{j+1}\left(t_{k}\right) \leq \bar{k}$. From $(4.5)$ at $t_{k}$ we have

$$
\int_{0}^{2 \pi} \sinh \left(k-M-\tilde{p}^{j}(s)\right)+\sinh \left(\tilde{p}^{j+1}(s)-\bar{k}-M\right) \leq 0 \quad \forall k \geq \bar{k} .
$$

Taking into account the summability of $\sinh \tilde{p}^{j}$, we get, sending $k$ to infinity, a contradiction by Levi's theorem. Thus $A_{k}^{j+1}$ has positive measure $\forall k$. Repeating the argument, we find $A_{k}^{j+N-1} \subset A_{k}^{j+N-2} \ldots \subset A_{k}^{j}, k \in \mathbb{N}$ sets of positive measure, such that

$$
\tilde{p}^{i}(t) \geq k \text { for } i=1 \ldots, N \text { and } k \in \mathbb{N}, t \in A_{k}^{j+N-1} \text {. }
$$


Since $\sum_{i=1}^{N} \tilde{p}^{i}(t) \equiv 0$, we arrive at a contradiction. Similarly, using (4.6), we can derive ess inf $\tilde{p}^{j}>-\infty \quad \forall j$.

Lemma 8. Let $\Psi, \Gamma$ satisfy (4.1). Then $\gamma^{j}-\gamma^{j-1} \in C^{0,1} \forall j$.

Proof. By Lemma 7 and (4.2), we see that $\Psi \in L^{\infty}$ and hence $L \Psi \in C^{0,1}$ (see (2.7)).

We first derive from (4.2) and (2.6)

$$
\begin{aligned}
\int_{0}^{\pi} \exp \left[\gamma^{j}-\gamma^{j-1}-L\left(\psi^{j}-\psi^{j-1}\right)\right](x, t-x)-\exp \left[\gamma^{j+1}-\gamma^{j}-L\left(\psi^{j+1}-\psi^{j}\right)\right](x, t-x) \mathrm{d} x \\
=\int_{0}^{\pi} \exp \left[\gamma^{j}-\gamma^{j-1}-L\left(\psi^{j}-\psi^{j-1}\right)\right](x, t+x)-\exp \left[\gamma^{j+1}-\gamma^{j}-L\left(\psi^{j+1}-\psi^{j}\right)\right](x, t+x) \mathrm{d} x
\end{aligned}
$$

for every $j$ and a.e. $t$. Thus, after writing $g^{j}=\exp \left[-L\left(\psi^{j}-\psi^{j-1}\right)\right] \in C^{0,1}$, we have, using previous notations,

$$
\begin{aligned}
\int_{0}^{\pi} g^{j}(x, t- & x) \exp \left[\tilde{p}^{j}(t)-\tilde{p}^{j}(t-2 x)\right]-g^{j}(x, t+x) \exp \left[\tilde{p}^{j}(t+2 x)-\tilde{p}^{j}(t)\right] \mathrm{d} x \\
& =\int_{0}^{\pi} g^{j+1}(x, t-x) \exp \left[\tilde{p}^{j+1}(t)-\tilde{p}^{j+1}(t-2 x)\right]-g^{j+1}(x, t+x) \exp \left[\tilde{p}^{j+1}(t+2 x)-\tilde{p}^{j+1}(t)\right] \mathrm{d} x
\end{aligned}
$$

(arabic@section.arabic@equation)

for every $j$ and a.e. $t$. Computing at $t+h, t$, and taking the difference, we see that

$$
\begin{aligned}
I^{j}(t, h):= & \int_{0}^{\pi} g^{j}(x, t+h-x) \exp \left[\tilde{p}^{j}(t+h)-\tilde{p}^{j}(t+h-2 x)\right]-g^{j}(x, t-x) \exp \left[\tilde{p}^{j}(t)-\tilde{p}^{j}(t-2 x)\right] \mathrm{d} x \\
& -\int_{0}^{\pi} g^{j}(x, t+h+x) \exp \left[\tilde{p}^{j}(t+h+2 x)-\tilde{p}^{j}(t+h)\right]-g^{j}(x, t+x) \exp \left[\tilde{p}^{j}(t+2 x)-\tilde{p}^{j}(t)\right] \mathrm{d} x \\
= & k_{1}^{j}(t, h)-k_{2}^{j}(t, h)
\end{aligned}
$$

is independent on $j$ for every fixed $h$ and a.e. $t$. Now,

$$
\begin{aligned}
k_{1}^{j}=\left[\mathrm{e}^{\tilde{p}^{j}(t+h)}-\mathrm{e}^{\tilde{p}^{j}(t)}\right] \int_{0}^{\pi} g^{j}(x, & t-x) \mathrm{e}^{-\tilde{p}^{j}(t-2 x)} \mathrm{d} x \\
& +\mathrm{e}^{\tilde{p}^{j}(t+h)}\left[\int_{0}^{\pi} g^{j}(x, t+h-x) \mathrm{e}^{-\tilde{p}^{j}(t+h-2 x)} \mathrm{d} x-\int_{0}^{\pi} g^{j}(x, t-x) \mathrm{e}^{-\tilde{p}^{j}(t-2 x)}\right] \mathrm{d} x .
\end{aligned}
$$

Since $\int_{0}^{\pi} g^{j}(x, t+h-x) \mathrm{e}^{-\tilde{p}^{j}(t+h-2 x)} \mathrm{d} x=\int_{-\frac{h}{2}}^{\pi-\frac{h}{2}} g^{j}\left(x+\frac{h}{2}, t-x+\frac{h}{2}\right) \mathrm{e}^{-\tilde{p}^{j}(s-2 x)} \mathrm{d} x$, we see that $\int_{0}^{\pi} g^{j}(x, t+$ $h-x) \mathrm{e}^{-\tilde{p}^{j}(t+h-2 x)} \mathrm{d} x-\int_{0}^{\pi} g^{j}(x, t-x) \mathrm{e}^{-\tilde{p}^{j}(t-2 x)} \mathrm{d} x=O(h)$, because $g^{j}$ is Lipschitz and $\tilde{p}^{j} \in L^{\infty}$, and hence

$$
k_{1}^{j}=c_{1}^{j}(t, h)\left[\tilde{p}^{j}(t+h)-\tilde{p}^{j}(t)\right]+O(h)
$$

where $c_{1}^{j}(t, h)=\int_{0}^{1} \mathrm{e}^{s \tilde{p}^{j}(t+h)+(1-s) \tilde{p}^{j}(t)} \mathrm{d} s \int_{0}^{\pi} g^{j}(x, t-x) \mathrm{e}^{-\tilde{p}^{j}(t-2 x)} \mathrm{d} x$ is positive, (essentially) bounded and bounded away from zero uniformly w.r. to $h$. Similarly

$$
k_{2}^{j}=-c_{2}^{j}\left[\tilde{p}^{j}(t+h)-\tilde{p}^{j}(t)\right]+O(h) .
$$

Hence

$$
I^{j}(t, h)=c^{j}\left(\tilde{p}^{j}(t+h)-\tilde{p}^{j}(t)\right)+O_{j}(h)
$$


where $c^{j}=c_{1}^{j}+c_{2}^{j}$. Since $I^{1}(t, h)=I^{2}(t, h)=\ldots=I^{N}(t, h)$ for every $h$ and a.e. $t$, then

$$
\tilde{p}^{j+1}(t+h)-\tilde{p}^{j+1}(t)=r_{j}\left[\tilde{p}^{j}(s+h)-\tilde{p}^{j}(s)\right]+O_{j}(h) \quad \text { a.e. } \quad \text { (arabic@section.arabic@equation) }
$$

where $r_{j}=r_{j}(t, h)=\frac{c^{j}}{c^{j+1}}$ are positive, bounded and bounded away from zero. Now, let

$$
A(M, h):=\left\{t: \tilde{p}^{1}(t+h)-\tilde{p}^{1}(t) \geq M|h|\right\} . \quad \text { (arabic@section.arabic@sequation) }
$$

It follows from (4.8) that, for a.e. $t \in A(M, h)$,

$$
\tilde{p}^{j+1}(t+h)-\tilde{p}^{j+1}(t) \geq r_{1} r_{2} \ldots r_{j} M|h|+O_{j}(h) \quad \text { for } j=1, \ldots, N-1 .
$$

Taking into account that $\sum_{j=1}^{N} \tilde{p}^{j} \equiv 0$, we obtain, for a.e. $t \in A(M, h)$,

$$
0 \geq\left(1+r_{1}+r_{1} r_{2}+\cdots+r_{1} r_{2} \ldots r_{N-1}\right) M|h|+O(|h|)
$$

and hence ess $\sup _{t}\left[\tilde{p}^{1}(t+h)-\tilde{p}^{1}(t)\right]=O(h)$. Similarly, ess $\inf _{t}\left[\tilde{p}^{1}(t+h)-\tilde{p}^{1}(t)\right]=O(h)$. By (4.8) it follows that ess $\sup _{t}\left|\tilde{p}^{j}(t+h)-\tilde{p}^{j}(t)\right|=O(h) \quad \forall j$, and hence $\tilde{p}^{j}, j=1, \ldots, N$, are (coincide a.e. with) Lipschitz continuous functions.

Notice that Lemma 8 and (4.2) imply $\Psi \in C^{0,1}$ and hence $L \Psi \in C^{1,1}$ by (2.8).

The final step is an iterative scheme that, thanks to Lemma 8 and what we have just observed, will imply $\Psi \in C^{\infty}$.

Proposition 9. Let $\Psi, \Gamma$ satisfy (4.1). Then, for $k=1,2, \ldots$

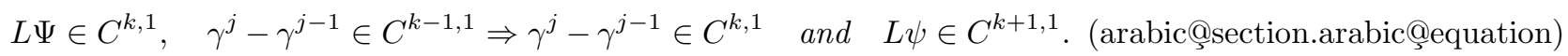

Proof. Let us discuss first in detail the case $k=1$. Notice first that $L \Psi \in C^{1,1} \Rightarrow g^{j} \in C^{1,1}$. Then, since $\tilde{p}^{j}$, being Lipschitz, has a (bounded) derivative almost everywhere, we can take the $t$ derivative in (4.7), to obtain that

$$
\begin{aligned}
J^{j}(t):= & \int_{0}^{\pi}\left[g_{t}^{j}(x, t-x) \mathrm{e}^{\tilde{p}^{j}(t)-\tilde{p}^{j}(t-2 x)}-g_{t}^{j}(x, t+x) \mathrm{e}^{\tilde{p}^{j}(t+2 x)-\tilde{p}^{j}(t)}\right] \mathrm{d} x \\
& +\int_{0}^{\pi} g^{j}(x, t-x) \mathrm{e}^{\tilde{p}^{j}(t)-\tilde{p}^{j}(t-2 x)}\left[\dot{\tilde{p}}^{j}(t)-\dot{\tilde{p}}^{j}(t-2 x)\right]-g^{j}(x, t+x) \mathrm{e}^{\tilde{p}^{j}(t+2 x)-\tilde{p}^{j}(t)}\left[\dot{\tilde{p}}^{j}(t+2 x)-\dot{\tilde{p}}^{j}(t)\right] \mathrm{d} x
\end{aligned}
$$

is independent on $j$, for a.e. $t$. Since $\dot{\tilde{p}}^{j}$ is bounded and $g_{t}^{j}$ is Lipschitz, we can write

$$
J^{j}=A^{j} \dot{\tilde{p}}^{j}+B^{j}
$$

with $A^{j}, B^{j}$ Lipschitz continuous, $A^{j}$ positive, bounded and bounded away from zero. Hence

$$
\dot{\tilde{p}}^{j+1}=\frac{A^{j}}{A^{j+1}} \dot{\tilde{p}}^{j}+C^{j}
$$

with $C^{j}$ Lipschitz. Thus, for any given $h \in \mathbb{R}$,

$$
\dot{\tilde{p}}^{j+1}(t+h)-\dot{\tilde{p}}^{j+1}(t)=\frac{A^{j}}{A^{j+1}}\left[\dot{\tilde{p}}^{j}(t+h)-\dot{\tilde{p}}^{j}(t)\right]+O(h) \quad \text { for a.e. } t .
$$

Since $\sum_{j=1}^{N} \dot{\tilde{p}}^{j} \equiv 0$, we conclude as in Lemma 8 that $\dot{\tilde{p}}^{j} \in C^{0,1}$. As above, $\tilde{p}^{j}, L \Psi \in C^{1,1} \Rightarrow \Psi \in C^{1,1} \Rightarrow L \Psi \in$ $C^{2,1}$. 
In the same way we can deal with $k \geq 2$ in (4.10). First observe that $g^{j} \in C^{k, 1}$ because $L \Psi \in C^{k, 1}$. Since $\tilde{p}^{j} \in C^{k-1,1}$ and hence $\left(\tilde{p}^{j}\right)^{(k)}$ exists as an $L^{\infty}$ function, we can take the $k$ th derivative in (4.7) to obtain that

$$
I_{k}^{j}(t):=\int_{0}^{\pi} \frac{\mathrm{d}^{k}}{\mathrm{~d} t^{k}}\left[g^{j}(x, t-x) \mathrm{e}^{\tilde{p}^{j}(t)-\tilde{p}^{j}(t-2 x)}-g^{j}(x, t+x) \mathrm{e}^{\tilde{p}^{j}(t+2 x)-\tilde{p}^{j}(t)}\right]
$$

is, for a.e. $t$, independent on $j$. As above, we can write

$$
I_{k}^{j}(t)=A_{k}^{j}(t)\left(\tilde{p}^{j}\right)^{(k)}+B_{k}^{j}(t)
$$

with $A_{k}^{j}, B_{k}^{j}$ Lipschtiz, $A_{k}^{j}$ positive, bounded and bounded away from zero. Again, this implies $\left(\tilde{p}^{j}\right)^{(k)} \in C^{0,1}$. From (4.2) we then derive that $\Psi \in C^{k, 1}$ and hence $L \Psi \in C^{k+1,1}$ by (2.8).

\section{Appendix}

Given $\xi \in \mathbb{R}^{N}$, let $[\xi]:=\max \left\{\xi^{1}-\xi^{N}, \xi^{2}-\xi^{1}, \ldots \xi^{N}-\xi^{N-1}\right\},|\xi|=\left(\sum_{j=1}^{N}\left|\xi^{j}\right|^{2}\right)^{\frac{1}{2}},|\xi|_{\infty}=\max _{i}\left|\xi^{i}\right|$ and denote $\mathbb{1}=(1,1, \ldots 1), c_{N}=\frac{2}{\sqrt{N}(N-1)}$. We have

Lemma A1. $[\xi] \geq \frac{2}{N-1}|\xi|_{\infty} \geq c_{N}|\xi| \quad \forall \xi \in \mathbb{1}^{\perp}$.

Proof. After writing $\xi^{j+k N}=\xi^{j}, j=1, \ldots N, k \in \mathbb{Z}$ (i.e. $\xi \in \mathbb{R}^{N}$ is identified with an $N$ periodic sequence), we have for any given $j \in\{1, \ldots, N\}$

$$
\begin{aligned}
& \xi^{j} \leq \xi^{j-1}+[\xi] \\
& \xi^{j} \leq \xi^{j-2}+2[\xi] \\
& \vdots \\
& \xi^{j} \leq \xi^{j-(N-1)}+(N-1)[\xi]
\end{aligned}
$$

and

$$
\begin{aligned}
& \xi^{j+1} \leq \xi^{j}+[\xi] \\
& \xi^{j+2} \leq \xi^{j}+2[\xi] \\
& \vdots \\
& \xi^{j+(N-1)} \leq \xi^{j}+(N-1)[\xi] .
\end{aligned}
$$

(arabic@ssection.arabic@̣equation)

Adding up the $N-1$ inequalities in (5.1) we get

$$
(N-1) \xi^{j} \leq \sum_{i \neq j} \xi^{i}+(1+2+(N-1))[\xi]
$$

Since $\sum_{i \neq j} \xi^{i}=-\xi^{j}$, we have

Similarly, from (5.2) we get

$$
N \xi^{j} \leq \frac{N(N-1)}{2}[\xi]
$$

$$
-\xi^{j} \leq \frac{N-1}{2}[\xi]
$$

Lemma A2. Let $f(\xi)=\exp \left(\xi^{1}-\xi^{N}\right)+\ldots+\exp \left(\xi^{N}-\xi^{N-1}\right)-N$. Then

(i) $\forall \alpha>0,\langle\nabla f(\xi), \xi\rangle \geq \alpha f(\xi) \quad \forall \xi \in \mathbb{1}^{\perp}$, with $|\xi| \geq \frac{\alpha+\sqrt{N \alpha}}{c_{N}}$;

(ii) $\langle\nabla f(\xi), \xi\rangle \geq c_{N}|\xi|\left[\mathrm{e}^{c_{N}|\xi|}-1\right] \quad \forall \xi \in \mathbb{1}^{\perp}$;

(iii) $\left(\mathrm{e}^{c_{N}|\xi|}-c_{N}|\xi|-1\right) \leq f(\xi) \leq N\left(\mathrm{e}^{\sqrt{2}|\xi|}-\sqrt{2}|\xi|-1\right) \forall \xi \in \mathbb{1}^{\perp}$; 
(iv) $\left\langle D^{2} f(\eta) \xi, \xi\right\rangle \geq c_{N}^{2} \mathrm{e}^{-\sqrt{2}|\eta|}|\xi|^{2}, \forall \eta \in \mathbb{R}^{N}, \forall \xi \in \mathbb{1}^{\perp}$.

Proof. (i)-(ii): Let $[\xi]=\xi^{j}-\xi^{j-1}$. Then

$$
\begin{aligned}
\langle\nabla f(\xi), \xi\rangle-\alpha f(\xi) & =\sum_{i \neq j}\left(\left(\xi^{i}-\xi^{i-1}\right)-\alpha\right) \mathrm{e}^{\xi^{i}-\xi^{i-i}}+([\xi]-\alpha) \mathrm{e}^{[\xi]}+\alpha N \\
& \geq \sum_{i \neq j}\left(\left(\xi^{i}-\xi^{i-1}\right)-\alpha\right) \mathrm{e}^{\alpha}+([\xi]-\alpha) \mathrm{e}^{[\xi]} .
\end{aligned}
$$

Since $\sum_{i=1}^{N}\left(\xi^{i}-\xi^{i-1}\right)=0$, we have

$$
\langle\nabla f(\xi), \xi\rangle-\alpha f(\xi) \geq-[[\xi]+(N-1) \alpha] \mathrm{e}^{\alpha}+([\xi]-\alpha) \mathrm{e}^{[\xi]} . \quad \text { (arabic@section.arabic@equation) }
$$

From

$$
(s-\alpha) \mathrm{e}^{s}-(s+(N-1) \alpha) \mathrm{e}^{\alpha} \geq \mathrm{e}^{\alpha}\left[(s-\alpha)^{2}-N \alpha\right] \quad \geq 0 \quad \text { if } s \geq \alpha+\sqrt{N \alpha}
$$

we see that $\langle\nabla f(\xi), \xi\rangle \geq \alpha f(\xi)$ if $[\xi] \geq \alpha+\sqrt{N \alpha}$, and hence (i) in view of Lemma A1.

Taking $\alpha=0$, in (5.3), we get (ii).

(iii) From (ii), $f(\xi)=\int_{0}^{1}\langle\nabla f(t \xi), \xi\rangle \mathrm{d} t \geq \int_{0}^{1} c_{N}|\xi|\left[\mathrm{e}^{c_{N} t|\xi|}-1\right] \mathrm{d} t=\mathrm{e}^{c_{N}|\xi|}-c_{N}|\xi|-1$.

As for the RHS inequality, we have

$$
f(\xi)=\sum_{i=1}^{N}\left(\mathrm{e}^{\xi^{i}-\xi^{i-1}}-1\right)=\sum_{\xi^{i}-\xi^{i-1}<0}\left[\mathrm{e}^{\xi^{i}-\xi^{i-1}}-\left(\xi^{i}-\xi^{i-1}\right)-1\right]+\sum_{\xi^{i}-\xi^{i-1}>0}\left[\mathrm{e}^{\xi^{i}-\xi^{i-1}}-\left(\xi^{i}-\xi^{i-1}\right)-1\right] .
$$

Since $\mathrm{e}^{s}-s-1$ is increasing in $[0, \infty)$ and $\left(\xi^{i}-\xi^{i-1}\right)^{2} \leq 2\left(\left|\xi^{i}\right|^{2}+\left|\xi^{i-1}\right|^{2}\right) \leq 2|\xi|^{2}$, while $\mathrm{e}^{s}-s-1 \leq \frac{s^{2}}{2}$ in $(-\infty, 0]$, we see that, if $p$ is the number of $i$ such that $\xi^{i}-\xi^{i-1}<0$, then

$$
f(\xi) \leq(N-p)\left[\mathrm{e}^{\sqrt{2}|\xi|}-\sqrt{2}|\xi|-1\right]+\sum_{\xi^{i}-\xi^{i-1}<0} \frac{\left(\xi^{i}-\xi^{i-1}\right)^{2}}{2} \leq(N-p)\left[\mathrm{e}^{\sqrt{2}|\xi|}-\sqrt{2}|\xi|-1\right]+\min \{p, 2\}|\xi|^{2}
$$

and the RHS inequality follows because

$$
\begin{gathered}
(N-p)\left[\mathrm{e}^{s}-s-1\right]+\min \{p, 2\} \frac{s^{2}}{2} \leq N\left(\mathrm{e}^{s}-s-1\right) \quad \forall s . \\
\text { (iv) }\left\langle D^{2} f(\eta) \xi, \xi\right)=\left.\frac{\mathrm{d}^{2}}{\mathrm{~d} t^{2}} f(\eta+t \xi)\right|_{t=0}=\sum_{j=1}^{N}\left(\xi^{j}-\xi^{j-1}\right)^{2} \mathrm{e}^{\eta^{j}-\eta^{j-1}} .
\end{gathered}
$$

Since $\min \left\{\eta^{1}-\eta^{N}, \ldots, \eta^{N}-\eta^{N-1}\right\} \geq-\sqrt{2}|\eta|$, we have,

$$
\left\langle D^{2} f(\eta) \xi, \xi\right\rangle \geq \mathrm{e}^{-\sqrt{2}|\eta|}[\xi]^{2} \geq c_{N}^{2} \mathrm{e}^{-2|\eta|}|\xi|^{2} .
$$

We now recall some basic facts on Orlicz spaces, used throughout the paper.

An Orlicz function (usually called $N$-functions, see [1]) is

$$
A(s)=\int_{0}^{s} a(t) \mathrm{d} t, \quad s \geq 0,
$$


where $a(0)=0, a \in c^{1}$ strictly increasing and satisfies $\lim _{s \rightarrow+\infty} \frac{a(s)}{s}=+\infty$. It's conjugate Orlicz function is given by

$$
A^{*}(s)=\sup _{t \geq 0}\{s t-A(t)\} .
$$

In the paper we have used $A(s)=\mathrm{e}^{s}-s-1$ for which $A^{*}(s)=(1+s) \log (1+s)-s$. $A^{*}$ enjoys the $\Delta_{2}$ (global) property:

$$
\left(\Delta_{2}\right) \quad \forall r>0 \quad \exists k(r)>0: \quad A^{*}(r s) \leq k(r) A^{*}(s) \quad \forall s \geq 0 .
$$

The set $K_{A}\left(\Omega_{n}\right)=K_{A}=\left\{\Phi \in L^{1}\left(\Omega_{n}, \mathbb{R}^{n}\right): \int_{\Omega_{n}} A(|\Phi|)<\infty\right\}$ is called the $A$-Orlicz class and its linear hull,

$$
L_{A}=\left\{\varphi: \lambda \varphi \in K_{A} \text { for some } \lambda>0\right\}
$$

is the Orlicz space. Endowed with the Orlicz-Luxemurg norm,

$$
\|\Phi\|_{A}=\inf \left\{k>0: \int A\left(\frac{|\Phi|}{k}\right) \leq 1\right\}
$$

the space $L_{A}$ becomes a Banach space.

Proposition A1 ([1] (pp. 232-240)). Let $A^{*}$ satisfy $\left(\Delta_{2}\right)$, then

(i) $K_{A^{*}}=L_{A^{*}}$.

(ii) $C_{0}^{\infty}\left(\Omega_{n}\right)$ is dense in $L_{A^{*}}$.

(iii) The dual space $L_{A^{*}}^{\prime}$ is isomorphic and homeomorphic to $L_{A}$.

Remark. The duality pairing between $L_{A^{*}}$ and $L_{A}$ is given by

$$
L_{A^{*}} \times L_{A} \ni(\Psi, \Phi) \rightarrow \int \Phi \Psi .
$$

Furthermore, we have from Hölder, (see [1] 237)

$$
\left|\int \Phi \Psi\right| \leq\|\Phi\|_{L_{A^{*}}}\|\Phi\|_{L_{A}}
$$

Proposition A2 ([7] (Th. 9.5)).

(i) $\|\Phi\|_{A} \leq 1 \Rightarrow \int_{\Omega_{n}} A(|\Phi|) \leq\|\Phi\|_{A},\|\Phi\|_{A}>1 \Rightarrow \int_{\Omega_{n}} A(|\Phi|) \geq\|\Phi\|_{A}$.

(ii) If $A$ satisfies $\left(\Delta_{2}\right)$, then

$$
\int_{\Omega_{n}} A(|\Phi|) \leq \sup _{t>0} \frac{A\left(\|\Psi\|_{A} t\right)}{A(t)}=k\left(\|\Phi\|_{A}\right) .
$$

Proposition A3 ([7] (Th. 4.6)). Let $\Omega_{n}$ have finite measure and $A^{*}$ satisfy $\left(\Delta_{2}\right)$. Let $\Phi_{n} \in L_{A}, \Phi_{n} \rightarrow \Phi$ in measure. If $\sup _{n}\left\|\Phi_{n}\right\|_{A}<\infty$, then $\Phi \in L_{A}$ and $\int \Phi_{n} \Psi \rightarrow \int \Phi \Psi \quad \forall \Psi \in L_{A^{*}}$.

Acknowledgements. Part of this work was done when the second author was visiting the Department of Mathematics, Rome 3, Italy. Part of this work was done when the first author was visiting TIFR Centre, Bangalore, India. They thank their respective hosts for the hospitality.

\section{REFERENCES}

[1] R.A. Adams, Sobolev Spaces. A.P (1975).

[2] V.I. Arnold, Proof of a Theorem of A.N. Kolmogorov on the invariance of quasi-periodic motions under small perturbations of the Hamiltonian. Russ. Math. Surv. 18 (1963) 9-36. 
3] H. Brezis and L. Nirenberg, Forced vibrations for a nonlinear wave equation. CPAM, XXXI(1) (1978) 1-30.

[4] H. Brezis, J.M. Coron and L. Nirenberg, Free Vibrations for a Nonlinear Wave Equation and a Theorem of P. Rabinowitz. CPAM, XXXIII (1980) 667-684.

[5] G. Friesecke and A.D. Wattis Jonathan, Existence Theorem for Solitary Waves on Lattices. Commun. Math. Phys. 161 (1994) 391-418.

[6] G. Iooss, Travelling waves in the Fermi-Pasta-Ulam lattice. Nonlinearity 13 (2000) 849-866.

[7] M.A. Krasnoselsky and Y.B. Rutitsky, Convex Functions and Orlicz Spaces. Internat. Monogr. Adv. Math. Phys. Hindustan Publishing Corpn., India (1962).

[8] H. Lovicarova', Periodic solutions of a weakly nonlinear wave equation in one dimension. Czechmath. J. 19 (1969) 324-342.

[9] J. Moser, On invariant curves of area-preserving mappings of an annulus. Nachr. Akad. Wiss. Göttingen, K1 2 (1962) 1.

[10] A.V. Mikhailov, Integrability of a Two-Dimensional Generalization of the Toda Chain. JETP Lett. 30 (1979) 414-413.

[11] L. Nirenberg, Variational Methods in nonlinear problems. M. Giaquinta Ed., Springer-Verlag, Lect. Notes Math. 1365 (1987).

[12] P.H. Rabinowitz, Periodic solutions of Hamiltonian Systems. Comm. Pure Appl. Math. 31 (1978) 157-184.

[13] B. Ruf and P.N. Srikanth, On periodic Motions of Lattices of Toda Type via Critical Point Theory. Arch. Ration. Mech. Anal. 126 (1994) 369-385.

[14] M. Toda, Theory of Nonlinear Lattices. Springer-Verlag (1989). 